\title{
Sobre las relaciones entre democracia y constitucionalismo
}

Constitutionalism and Democracy

M. Lourdes Santos Pérez

Universidad de Salamanca lula@usal.es

DOI: http://doi.org/10.15366/bp2019.20.005 Bajo Palabra. II Época. No20. Pgs: 87-106 


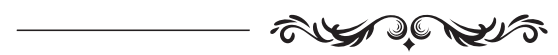

\section{Resumen}

La organización del poder político en una sociedad democrática no está exenta de dificultades. Así, el fenómeno conocido como constitucionalismo ha traído a primer plano la discusión a propósito de si este y democracia son principios opuestos sobre los que una teoría política sensata debe intentar alcanzar un compromiso, o son por el contrario principios complementarios que tienen un origen común. Ronald Dworkin, uno de los filósofos del derecho más importante de las últimas décadas, ofrece una reflexión sugerente en torno a esta cuestión. Esta indagación no sólo resulta de interés en sí misma considerada sino que el modo como la articula arroja luz a propósito de la comprensión del derecho como fenómeno institucional.

Palabras Clave: Derecho, Institución, Democracia, Constitucionalismo, Liberalismo. Dworkin

\section{Abstract}

The organization of political power in a democratic society is not without its difficulties. Thus, the phenomenon known as constitutionalism has brought to the fore the discussion about whether this and democracy are opposite principles on which a sensible political theory should attempt to reach a compromise or are on the contrary complementary principles that have a common origin. Ronald Dworkin, one of the most important philosophers of law in recent decades, offers a suggestive reflection on this issue. This inquiry is not only of interest considered, but the way in which the article sheds light on the understanding of law as an institutional phenomenon.

Keywords: Law, Institution, Democracy, Constitutionalism, Liberalism, Dworkin 
Me gustaría, antes Que NADA, advertir que, aunque lo que se analizará en las páginas que siguen pertenece inequívocamente al campo de la filosofía política, el pensamiento de Dworkin envuelve fuertes pretensiones holistas. En efecto, éste, tomando pie en un ensayo de Berlin ${ }^{1}$, se identificó con el modo de pensar característico de los "erizos". Berlin, en este sentido, distinguió dos tipos de pensadores. Por un lado, aquellos que tienen una visión dispersa y múltiple de la realidad, que perseguirían fines diferentes, en ocasiones contradictorios entre sí, sin pretender integrarlos en una única visión globalizadora. Estos se conducirían como el "zorro" de Arquíloco, que "sabe muchas cosas". Por otro lado, están aquellos que aspiran a poder articular una visión del mundo coherente a partir de un principio rector o de un conjunto coherente de principios y pretenden organizar su vida con unidad y armonía. Estos últimos se comportarían más bien como el "erizo" de Arquíloco, que solo sabe una cosa, pero esa es "muy importante".

Así las cosas, la pretensión de Dworkin, revelada ya desde sus primeros escritos, fue dotar de coherencia e integridad a toda la estructura de pensamiento. Específicamente, si tuviésemos que ponernos a la tarea de examinar toda su obra, la, llamémosla así, secuencia "teoría del derecho-teoría política-teoría ética" sería su modo natural de exposición.

Expresándolo de manera apretada, la teoría del Derecho de Dworkin, tal y como se formula en Taking Rights Seriously ${ }^{2}$ y, sobre todo, en Law's Empire ${ }^{3}$ está dominada por intenciones políticas. Singularmente, su concepción del derecho como "integridad" establece una conexión necesaria entre teoría del derecho y teoría política. En efecto, la integridad exige a la comunidad actuar aplicando de forma consistente los principios de una filosofía política definida, aunque esa filosofía no sea compartida en todos sus extremos por todos sus miembros. Dicha concepción, singularmente, aplicada al campo del derecho constitucional ${ }^{4}$, abre las puertas a una reflexión sobre

\footnotetext{
${ }^{1}$ Berlin, The Hedgehog and the Fox, Weidenfeld and Nicolson Ldt., 1953.

2 Dworkin, Taking Rights Seriously, London, Duckworth, 1981.

3 Dworkin, Law's Empire, London, Fontana Press, 1986.

${ }^{4}$ La concepción interpretativa del derecho como integridad, aplicada al campo del derecho constitucional, conduce a lo que Dworkin denomina "una lectura moral de la constitución”. Que la interpretación jurídica de la constitución requiere una teoría política o lectura moral significa que, cuando un juez se enfrenta a un caso
} 
algunos temas centrales de la teoría del Estado constitucional, como la significación del constitucionalismo y la justificación de los tribunales constitucionales, que analizaremos en las páginas siguientes.

Ahora bien, antes de entrar en esta temática, me gustaría remarcar que, en donde se revela de forma más significativa la importancia que tiene la idea de integridad para la concepción dworkiniana del derecho, es en la aplicación judicial de este. Por qué adoptar la perspectiva del juez como clave para la comprensión del derecho es un punto que merece especial atención.

La práctica jurídica es una práctica extraordinariamente compleja, en la que se entrecruzan las perspectivas de multitud de actores. Ahora bien que Dworkin sitúe al juez en el centro de la teoría obedece no (solo), como a menudo se ha alegado, a las peculiaridades del sistema jurídico angloamericano desde las que elabora su teoría, sino más bien a la singular posición que ocupan los jueces en el derecho como sistema normativo institucionalizado.

En el proceso de definición y especificación del derecho como sistema normativo $^{5}$, ha sido Raz ${ }^{6}$ quien ha desarrollado de forma más directa la tesis de que el derecho es un sistema normativo institucionalizado. El hecho de que el derecho sea un sistema normativo institucionalizado implica no sólo que es un sistema que crea y regula instituciones, sino también que sus propias normas son creadas y aplicadas por instituciones. Ahora bien, dentro del entramado de instituciones encargadas del proceso de reproducción del sistema, los tribunales ocupan un papel destacado. Raz los denomina "órganos primarios" porque en su existencia cifra la caracterización de un sistema normativo como sistema institucionalizado. Estos órganos tienen encomendada la función peculiar y específica que conocemos como función jurisdiccional.

Ahora bien, como sugiere la propia expresión, juris-dictio es una forma peculiar de aplicación del derecho que incluye una declaración sobre cuál es el derecho

que requiere interpretar algunos contenidos del texto constitucional, en concreto algunas de las disposiciones recogidas bajo la rúbrica genérica Bill of Rights, es inevitable incurrir en apreciaciones y juicios de valor políticos y morales. La razón para proceder de ese modo es que ese conjunto de disposiciones que declaran derechos negativos han sido redactados es un lenguaje deliberadamente abstracto con inequívocas connotaciones de carácter moral. La lectura moral, en consecuencia, propone que "todos nosotros, jueces, abogados y ciudadanos, interpretemos y apliquemos esa cláusulas abstractas en base al entendimiento de que invocan principios morales de decencia y justicia políticas”. Dworkin, Freedom's Law. The Moral Reading of American Constitution, Oxford, Oxford University Press, I 996

5 Desde Kelsen se viene afirmando entre los teóricos del derecho que la clave para descifrar el concepto de derecho no está en la noción de norma sino en la de sistema normativo, entre otras razones porque lo que caracteriza a una norma como jurídica, y además como una norma jurídica vigente, no es ni su contenido ni su forma, sino su pertenencia a un sistema normativo que tiene ciertos rasgos característicos.

${ }^{6}$ Raz, Practical Reason and Norms, Princeton, Princeton University Press, 1990. 
vigente. Naturalmente, no sólo los jueces, sino también los abogados cuando aconsejan a sus clientes, los científicos del derecho cuando analizan aspectos de este, incluso nosotros mismos cuando juzgamos sobre nuestra propia situación jurídica y la de otros, formulamos declaraciones sobre el derecho. Como cualquier declaración, esas declaraciones sobre el derecho vigente pueden ser discutidas desde el punto de vista de la verdad. Lo distintivo de las declaraciones de los jueces está, frente a todas los demás, en que ellas son vinculantes, es decir, son normas que obligan y surten efectos dentro del sistema, y que lo son incluso si son erróneas. Mientras los juicios emitidos por los abogados, los litigantes, los científicos del derecho, etc., son opiniones, mejor o peor fundadas, pero opiniones a fin de cuentas, los juicios de los jueces son sentencias, esto es, pronunciamientos definitivos que producen efectos dentro del sistema. En este sentido, las diversas argumentaciones que se producen en el espacio jurídico (cuando el abogado asesora a su cliente, o cuando el científico del derecho explora las consecuencias de una determinada regla, o cuando nos instruimos unos a otros sobre nuestras obligaciones legales) tienen como modelo la argumentación judicial. Que la teoría dworkiniana del derecho esté en gran medida construida desde la perspectiva del juez se explica, pues, por la naturaleza misma del fenómeno objeto de la teoría.

\section{II}

Al pRoblema de las SUpuestas tensiones existentes entre las ideas de democracia y constitucionalismo, Ronald Dworkin, uno de los filósofos del derecho más importante de las últimas décadas, dedicó su atención. Voy a tomar pie en sus escritos Equality, Democracy and Constitution, Constitutionalism and Democracy, la «Introducción» de Freedom's Law, «Reflections on Fidelity» y «The Partnership Conception of Democracy» para exponer su posición en este punto.

Como es sabido, uno de los rasgos característicos del moderno Estado constitucional es estar organizado con arreglo a un sistema jurídico que incorpora el reconocimiento y la protección constitucional de unos derechos individuales que operarían como límites materiales frente al poder de los gobiernos. La elevación de las viejas declaraciones de derechos al rango de normas jurídicas superiores conducía de forma natural a la aparición de mecanismos de control de la constitucionalidad de las leyes. Pues bien, este fenómeno, que simplificadamente se denomina constitucionalismo, ha empezado a ser, a pesar de su popularidad, objeto de fuertes críticas que insisten en que subvierte o compromete la democracia. Si una constitución prohíbe al legislador, democráticamente elegido, aprobar por ejemplo una ley 
que restrinja el núcleo central de la libertad de expresión, o que cercene los derechos de una minoría étnica, eso significa una disminución del derecho democrático que la mayoría tiene para promulgar las leyes que desean. ¿Constitucionalismo y democracia son principios opuestos sobre los que una teoría política sensata debe intentar alcanzar un compromiso, o son por el contrario principios complementarios que tienen un origen común?

Planteadas así la cosas, Dworkin, en un intento de interpretar la idea popular de que la democracia es la forma de gobierno del pueblo, defenderá una concepción que él denomina comunal [de la democracia], que ofrece en polémica frente a una visión meramente mayorista, muy extendida.

El punto de partida de su argumentación es la distinción entre dos formas de acción colectiva. Como cualquier otra forma de gobierno, la democracia envuelve algún tipo de acción colectiva: supone que un grupo de personas hace cosas que ninguna de ellas hace o puede hacer por sí sola. En particular, de una democracia decimos que es un régimen en el que gobierna el pueblo. Ahora bien, es posible interpretar de dos maneras diferentes esta acción colectiva o de grupo: estadística y comunalmente. Una acción es estadísticamente colectiva cuando lo que el grupo hace es sólo función de lo que miembros individuales del grupo hacen por sí solos, sin que en ello esté envuelto ningún sentido de estar haciendo algo como grupo. Así, por ejemplo, cuando hablamos de que el pueblo español prefiere veranear en la playa, "pueblo" es una mera abreviatura lingüística, y el hecho de que mayoritariamente los españoles tengan esa preferencia es un mero dato estadístico. Por el contrario, la acción es comunalmente colectiva cuando requiere que los individuos asuman la existencia del grupo como una entidad o fenómeno separado; por ejemplo, cuando decimos que una orquesta interpreta una sinfonía, no atribuimos la ejecución a una suma discreta de personas sino a esa colectividad que denominamos "orquesta".

A estas dos formas diferentes de acción colectiva podemos asociar dos formas diferentes de interpretar la idea de democracia como una forma de gobierno del pueblo: (1) según una interpretación estadística, en una democracia las decisiones políticas son hechas en consonancia con una función mayoritaria de los votos, decisiones o deseos de ciudadanos individuales tomados uno a uno; (2) según una interpretación comunal, en una democracia las decisiones políticas se imputan a una entidad diferente de la mera suma de individuos, el pueblo como tal ${ }^{7}$.

\footnotetext{
${ }^{7}$ Dworkin, «Equality, Democracy, and Constitution: We the People in Court», Alberta Law Review, 28, I990, p. 330. La misma distinción aparece desarrollada en Dworkin, «Constitutionalism and Democracy», European Journal of Philosophy, 3, I995, p. 3 sigs.; también en Dworkin, Freedom's Law. The Moral Reading of American Constitution, op. cit. p. 19 sigs.; finalmente en Dworkin, «The Partnership Conception of Democracy», California Law Review, 86, 1998.
} 
Una concepción estadística se ve en dificultades para explicar los límites característicos del constitucionalismo. Algunas limitaciones constitucionales pueden justificarse porque crean las condiciones que hacen posible la formación de la voluntad de una mayoría. Pero es obvio que no todas las limitaciones propias de un Estado constitucional pueden justificarse por esta vía. Por eso, para una visión estadística de la democracia, el constitucionalismo, que impone restricciones a lo que puede hacer y decidir una mayoría, es un principio limitante de la democracia, tal vez sensato dentro de una teoría política realista, pero en todo caso un principio en competencia con el principio democrático. Frente a ello Dworkin intenta defender que, interpretada la idea de democracia dentro de una concepción comunal, la existencia de límites constitucionales no sólo no supone un obstáculo para la democracia sino que forma parte de las condiciones de posibilidad de la misma:

la acción comunal colectiva es posible sólo si los miembros de la comunidad comparten ciertos ideales; si es así, el mantenimiento de esos ideales mediante restricciones impuestas a la decisión de la mayoría sería en sí mismo una forma de estructurar la democracia más que de cualificarla o socavarla ${ }^{8}$.

Para seguir avanzando en la comprensión de la interpretación comunal de la idea de democracia, hay que despejar un posible malentendido que podría suscitarse. En efecto, podría pensarse que esta concepción comunal de la democracia, que hace al "pueblo como tal" protagonista de las funciones de gobierno, envuelve, en el mejor de los casos, una mitificación de la colectividad, análoga a la mitificación del Estado en la filosofía hegeliana, cuando no una justificación de regímenes totalitarios sobre la base de que el estado es más importante que el individuo. Para mostrar que es posible una concepción comunal libre de esas implicaciones, Dworkin articula una nueva distinción entre lo que llama dos formas diferentes de interpretar la acción política comunal: la acción comunal puede ser integrada, o alternativamente, monolítica. Pero esta distinción requiere una breve aclaración.

Siempre que se actúa de forma consciente se hacen dos tipos de presunciones sobre la unidad de acción que se halla en juego. (1) Por una parte se presume una determinada unidad de responsabilidad. En la mayor parte de los casos se atribuye la responsabilidad a los individuos; y esto ocurre así en los casos de acción colectiva estadística. Pero en otros supuestos, es al grupo al que se atribuye responsabilidad; por ejemplo, cuando los alemanes se sienten responsables de los crímenes perpetrados por los nazis, incluso si no participaron en ellos. (2) Por otra parte, se presume además una unidad de juicio. Aunque en la mayor parte de las ocasiones se

${ }^{8}$ Dworkin, «Equality, Democracy, and Constitution: We the People in Court», op. cit., p. 330. 
considera al individuo como la unidad de juicio, algunas personas entienden que la unidad básica de juicio es [y/o debe ser] el grupo, porque es la cultura del grupo la que configura las creencias de los individuos y, por consiguiente, su juicio; eso explicaría que un alemán nonagenario no sintiera vergüenza por su participación en las atrocidades cometidas por los nazis, atribuyéndolas a una cultura colectiva que las había abrazado como triunfos históricos.

Esta distinción permite ahora completar la clasificación inicial de las formas de acción colectiva en el sentido siguiente: al lado de la acción colectiva estadística, donde los individuos son la unidad de acción tanto de responsabilidad como de juicio, la acción colectiva comunal puede interpretarse de dos maneras distintas: como una acción integrada, en cuyo caso los participantes conforman una unidad colectiva de responsabilidad pero no de juicio o, alternativamente, como una acción monolítica, en cuyo caso es colectiva tanto la unidad de responsabilidad como la unidad de juicio. La posición de Dworkin es que una democracia genuina presupone una forma de acción política que además de comunal es integrada:

En una democracia genuina, el pueblo no gobierna estadísticamente sino comunalmente. Los ciudadanos tratan a su nación como una unidad colectiva de responsabilidad, lo que significa que ellos, como ciudadanos, comparten responsabilidad derivada por todo lo que su gobierno, actuando oficialmente, haga. Pero aunque el pueblo forma una unidad de responsabilidad diferenciada, no forma una unidad colectiva de juicio. En una democracia comunal, cada ciudadano insiste en que sus convicciones políticas son en un sentido muy importante un asunto propio, que es responsabilidad de uno mismo discernir aquello que se requiere que la nación haga bien, si lo ha hecho y en qué grado ha tenido éxito?

La estructura de una constitución democrática entendida así difiere de la de una concepción estadística: además de instituciones que articulen el poder y las funciones de los distintos cuerpos del gobierno se necesitan instituciones que «alimenten la pareja de actitudes democráticas que se necesita: responsabilidad colectiva y juicio individual $»^{10}$

Una concepción comunal de la democracia requiere una constitución robusta que asegure las condiciones que ha de cumplir una asociación política para poder ser considerada una verdadera comunidad. Esas condiciones son de dos tipos: estructurales y relacionales ${ }^{11}$.

${ }^{9}$ Dworkin, «Equality, Democracy, and Constitution: We the People in Court», op. cit., p. 337.

10 Ibidem.

${ }^{11}$ En rigor, Dworkin sólo menciona en «Equality, Democracy and Constitution» el segundo grupo de condiciones aunque sin designarlas con el nombre de relacionales. La distinción entre condiciones estructurales y condiciones relacionales aparece en Dworkin, Freedom's Law, op. cit., p. 24. 
Son condiciones estructurales aquéllas que describen el carácter que debe tener una comunidad para que pueda reconocerse como verdadera asociación política. Entre ellas, Dworkin menciona de pasada la de disponer de un territorio estable, compartir una historia, una lengua común, un conjunto de valores, etc., condiciones en cuyo análisis Dworkin no tiene interés en detenerse. Más interés presta a las condiciones relacionales, que describen el trato que una comunidad debe dispensar a sus individuos para que puedan sentirse parte de ella, como «miembros morales». En concreto, Dworkin señala tres: una condición de participación, una condición de consideración (stake) y una condición de independencia ${ }^{12}$. Para que una asociación política pueda contar como una verdadera comunidad tiene que estar articulada de modo que cada individuo tenga una participación en el colectivo, una consideración en él, y al mismo tiempo una independencia como agente moral respecto del colectivo. Un breve examen muestra de qué modo las instituciones políticas que asociamos a una constitución democrática pueden justificarse como presupuestos estructurales necesarios para crear y mantener un "agente comunal integrado, el pueblo». ${ }^{13}$

a) La condición o principio de participación establece con carácter general que en una democracia se debe conceder a todas las personas la oportunidad de influir (make a difference) en las decisiones políticas, sin que la fuerza de ese papel pueda estar limitada por presunciones sobre su talento o capacidad. ${ }^{14}$ Este principio es suficiente para explicar por qué se asocia la democracia con el sufragio universal, con la idea de una persona un voto y con la idea de que los cargos políticos están en principio abiertos a todos [aunque en la configuración concreta de estas instituciones juega un cierto papel la historia de la comunidad, y por esta razón pueden admitirse desviaciones]. Finalmente, el principio de participación explica también por qué las libertades políticas, como la libertad de expresión y de manifestación, forman parte de la democracia sin necesidad de recurrir a la problemática idea de una igualdad de influencia: desde el punto de vista de una interpretación comunal de la democracia, estas libertades requieren que cada individuo pueda influir, no que su influencia tenga que ser igual.

\footnotetext{
${ }_{12}$ Dworkin, "Constitutionalism and Democracy», op. cit., pp. 4-5 y Dworkin, Freedom's Law, op. cit., p. 24.

13 Dworkin, «Equality, Democracy, and Constitution: We the People in Court», op. cit., p. 337.

${ }^{14}$ Una precisión. La primera parte del enunciado [a saber, que cada uno ha de tener un papel en la acción colectiva] vale para todas las formas de unidad colectiva, mientras que la segunda [a saber, que ese papel no puede ser más o menos fuerte en virtud del talento de cada uno] es característica de las formas de acción comunal organizadas democráticamente, pero no de todas las formas de acción comunal: no regiría, por ejemplo, en el caso de una orquesta.
} 
b) Por su parte, el principio de la consideración (stake) establece que en una democracia interpretada comunalmente las decisiones colectivas deben reflejar igual solicitud por los intereses de todos sus miembros. Así, una democracia que distribuyera los recursos de forma poco equitativa no sólo estaría actuando de forma injusta, sino también de forma no-democrática ${ }^{15}$. Ahora bien, esta definición del principio de la consideración suscita inmediatamente la pregunta de si, al disolver la línea de demarcación entre aspectos procedimentales y substantivos de la justicia, no se está bloqueando la distinción entre la justicia y los demás valores políticos. En el marco de una concepción estadística de la democracia parece que no hay dificultad alguna en decir que una decisión democrática puede ser injusta. Pero desde el momento en que, como ocurre con una concepción comunal, la idea de democracia se extiende más allá de los aspectos puramente procedimentales relativos a los procesos de decisión, parece que se impide esta forma de hablar, que sin embargo parece útil y con sentido. En suma, el principle of stake parece hacer de la idea de democracia «un agujero negro en el que colapsan todas las demás virtudes políticas» ${ }^{16}$. Para evitar esto, Dworkin propone la adopción de una versión débil del principio que exigiría, no que la comunidad consiga el mejor entendimiento de lo que requiere el principio abstracto de igual solicitud, sino sólo que acepte la idea como una exigencia abstracta. De este modo, se puede seguir hablando de democracia incluso cuando el proceso de decisión política produce decisiones injustas, pero con un límite: «un sistema político con igualdad de voto en el que la mayoría distribuye todo para ella, sin atender en modo alguno a la suerte de alguna minoría racial o de otro tipo, contará, desde una concepción comunal, no como una democracia injusta sino como un sistema no democrático» ${ }^{17}$.

c) El principio de independencia reconoce a los miembros de la comunidad su condición de agentes morales, responsables de su propio juicio moral. Si el principio de la consideración es fundamental para pasar a una democracia comunal, esto es, para pasar a una forma de acción colectiva en la que "el pueblo" se entienda más comunal que estadísticamente, el principio de independencia es necesario para entender una democracia comunal como una forma de acción política [comunal] integrada y no

15 Dworkin lo expresa recurriendo a una vieja fórmula: «la concepción comunal une justicia procedimental y substantiva insistiendo en que democracia significa gobierno por el pueblo y también para el pueblo» (Dworkin, «Equality, Democracy, and Constitution: We the People in Court», op. cit., p. 339). Las cursivas son mías.

${ }^{16}$ Ibidem.

${ }^{17}$ Ibidem. 
monolítica. ${ }^{18}$ El principio de independencia «insiste en que un gobierno democrático no tiene que dictar qué deben pensar sus ciudadanos en cuestiones de juicio moral o ético, sino que, por el contrario, tiene que limitarse a proporcionar un conjunto de circunstancias que les animen a llegar a creencias sobre esas cuestiones a través de una convicción reflexiva y en último término individual» ${ }^{19}$.

Consecuente con esta definición, este principio prohíbe medidas dirigidas a intentar moldear las convicciones personales de la gente. Por lo demás, tiene importantes consecuencias estructurales para la democracia: se suma, para empezar, al principio de participación para tratar las libertades políticas como estructurales para la democracia; insiste, además, en asignar un lugar estructural a las garantías constitucionales de la libertad de expresión, asociación y religión; finalmente, sanciona alguna forma de tolerancia liberal frente a conductas poco ortodoxas como una condición de la democracia ${ }^{20}$.

Frente a la concepción crudamente mayoritarista, que ve en la democracia un simple procedimiento de decisión por mayoría entre individuos independientes embarcados en un proceso competitivo en persecución de sus propios intereses, la concepción comunal aboca a una partnership conception ${ }^{21}$. Una democracia genuina es una forma de organización política en la que las funciones de gobierno son desempeñadas por el pueblo entendido como una forma de asociación colectiva (partnership) donde cada persona es un miembro moral y como tal parte activa (partner) en una empresa colectiva de autogobierno. Desde luego este ideal político puede desarrollarse institucionalmente de formas diferentes y en diferentes grados. Pero no hay un test único al que podamos recurrir para comprobar de una forma sencilla el grado de éxito que ha tenido una sociedad que intenta desarrollar esta forma de asociación política. Más bien hay que poner en juego un conjunto complejo de ideales que, según Dworkin, operarían en tres dimensiones: a saber, (1) la soberanía popular, (2) la igualdad de los ciudadanos y (3) el discurso democrático ${ }^{22}$. En esas tres dimensiones la partnership conception difiere de la concepción mayoritarista.

\footnotetext{
18 "La independencia de juicio [...] es una condición estructural de pertenencia a una comunidad integrada» (Dworkin, "Equality, Democracy, and Constitution: We the People in Court», op.cit., p. 341).

19 Dworkin, «Equality, Democracy, and Constitution: We the People in Court», op. cit., p. 340.

${ }^{20}$ Dworkin señala que si la democracia se entiende estadísticamente, entonces la tolerancia liberal sólo se puede defender con argumentos de justicia (Dworkin, «Equality, Democracy, and Constitution: We the People in Court", op. cit., pp. 341-2).

${ }^{21}$ Dworkin, Sovereign Virtue. The Theory and Practice of Equality, Cambridge, Mass., Harvard University Press, 2000, p. 358. La misma idea está presente en Dworkin, Freedom's Law, op. cit., p. 17, aunque aquí prefiere hablar de una "concepción constitucional de democracia».

22 Dworkin, Sovereign Virtue, op. cit., p. 362 sigs.
} 
(1) Para la partnership democracy, la soberanía popular es el tipo de relación que se establece entre las autoridades y el pueblo entendido como asociación de ciudadanos libres e iguales. La realización del ideal de la soberanía popular exige, entre otras cosas, abolición de privilegios derivados de la herencia, la casta, etc. Para la concepción mayoritarista de democracia, la soberanía popular más que una relación entre los poderes públicos y el pueblo es la clase de poder que ostenta una mayoría para regir los destinos de todos.

(2) Desde la segunda de las dimensiones, la partnership democracy demanda la articulación de procedimientos que aseguren a todos los ciudadanos el mismo poder para votar y hablar en los distintos procesos en que se han de tomar las decisiones de gobierno y configurar la cultura y opinión públicas. En este sentido apuesta por la idea de sufragio universal, sin que eso signifique que cada ciudadano deba tener la misma influencia sobre los otros a menos que la "fuente" de esa desigual influencia se deba a alguna forma de injusticia. También la concepción mayoritarista apoya el sufragio universal pero por una razón algo diferente: porque éste es el único medio para poder saber qué es lo que quiere la mayoría.

(3) Finalmente, la partnership democracy demanda desde la tercera de las dimensiones un contexto público de deliberación convenientemente articulado [por ejemplo, libre de censura], donde a cada ciudadano se le conceda la misma oportunidad de exponer sus razones para poder así tratar de influir en los otros antes de tomar una decisión común en un determinado sentido: «la democracia no puede proporcionar una forma genuina de autogobierno si los ciudadanos no son capaces de hablar a la comunidad en una estructura y en un clima que anima a prestar atención a los méritos de lo que ellos dicen» ${ }^{23}$.

\section{III}

Hasta aquí la exposición de la teoría de Dworkin sobre los presupuestos fundamentales del Estado constitucional democrático. Con dos advertencias. La primera es que aquélla se ve dificultada por una forma de organizar la argumentación que es muy característica del autor. Dworkin se conduce con soltura no sólo definiendo los problemas sino también construyendo a sus posibles adversarios y elaborando

${ }^{23}$ Dworkin, Sovereign Virtue, op. cit., p. 365. 
un aparato terminológico y conceptual original. Aunque a lo largo de la exposición intento aclarar los puntos más oscuros y situar debidamente los argumentos, no siempre he podido sustraerme a las peculiaridades casi idiosincrásicas de la escritura del autor. La segunda es que dicha reflexión forma parte, ya se ha dicho, de un proyecto ciertamente ambicioso donde el autor buscó organizar en un esquema teórico coherente exigencias de libertad, igualdad y eficiencia al amparo de un ideario netamente liberal, manteniendo la congruencia con los presupuestos básicos del Estado constitucional democrático.

Detenernos en esta segunda cuestión excede los límites del presente trabajo. En todo caso, voy a avanzar muy sumariamente cuáles son los criterios desde los que se debería proceder a evaluar críticamente dicho proyecto y, específicamente, su defensa del ideario democrático.

Dworkin, que se proclama a sí mismo liberal, acomete una interesante reflexión sobre el estado actual del liberalismo ${ }^{24}$. De acuerdo con su diagnóstico, éste aparece sumido en una profunda crisis de identidad que se desenvuelve en dos planos diferentes. Por un lado, en el marco de las discusiones políticas domésticas, a menudo hay dificultades para identificar un programa político como distintivamente liberal, porque no está claro cuáles son los ingredientes y componentes fundamentales del liberalismo, su núcleo esencial. Por otro lado, el liberalismo ha perdido buena parte del atractivo que en otro momento tuvo, porque no se han explorado debidamente sus fundamentos más profundos, sus vínculos con otras ramas del conocimiento, en particular con la ética.

Pues bien, para salir de esta crisis de identidad, el autor se propone seguir una estrategia doble: por una parte, identificar los elementos fundamentales del liberalismo, la estructura básica de principios de una concepción distintivamente liberal, los "fundamentos políticos" de fondo en que se apoyan propuestas políticas específicamente liberales; por otra, investigar la fundamentación filosófica de semejante concepción, sus "fundamentos filosóficos", en particular mostrar que esos principios tienen una conexión sólida con una concepción ética atractiva.

Así las cosas, la reflexión de Dworkin sobre las relaciones entre constitucionalismo y democracia forma parte de la indagación que éste ha hecho en torno a los denominados fundamentos políticos del liberalismo. En este sentido, dicha reflexión parece que cumpliría con ciertas condiciones ${ }^{25}$ que cualquier concepción política que se arrogue como constitutiva del ideario liberal (y no meramente derivada) debería cumplir.

\footnotetext{
${ }^{24}$ Tal vez, no está de más recordar que, lo que ha terminado considerándose pensamiento liberal, es el resultado de la acumulación histórica de ingredientes procedentes de una cierta variedad de doctrinas como son el liberalismo clásico, el utilitarismo, el socialismo y otros procedentes de la historia de la ilustración.

25 Dworkin, A Matter of Principle, Londres, Harvard University Press, 1985, p.186 ss.
} 
De este modo, se tendría en primer lugar que verificar si cumple con la condición de "completud". Siguiendo a Dworkin, ésta exige que la teoría permita dar cuenta del conjunto de causas indiscutiblemente consideradas liberales. El autor, en este punto, insiste en que su teoría sobre el núcleo básico del liberalismo ha de desarrollarse en dimensiones diversas; entre otras, en la esfera política. De este modo, articula una forma democrática de gobierno con trazas republicanas, al tiempo que reivindica derechos típicamente liberales, como el derecho a la libertad de expresión, la prohibición de la censura y los derechos procesales a los que considera límites al poder de las mayorías.

En segundo lugar, se refiere a una exigencia de "distinción". La teoría, en este sentido, debe ofrecer un criterio nítido de diferenciación respecto de otros idearios, particularmente del ideario conservador. Aquí Dworkin insiste en que el desacuerdo entre liberales y conservadores no reside en el rechazo o la preferencia por la democracia como mecanismo distributivo del poder político, pues ambos la aprueban, sino en las razones y el alcance de dicha aceptación. El liberal justifica esta institución por razones igualitarias; y son precisamente consideraciones igualitarias las que le inducen a defender la interposición de mecanismos correctivos diferentes de los que defienden los conservadores apoyándose en consideraciones moralistas. En palabras del autor, las diferencias radican en la clase de derechos que unos y otros estiman necesarios a fin de corregir las imperfecciones que pudiera generar el sistema democrático.

De manera que no parece que la concepción de Dworkin sobre la democracia falle en su intento de imponer un programa que pretenda valer como reconstrucción del liberalismo. Todavía, sin embargo, falta por verificar si esa teoría dispone de unos fundamentos sólidos, cuya naturaleza el autor precisa cualificándolos como "éticos"26.

Esta cualificación de la tarea de fundamentar el liberalismo es interesante porque, como es notorio, una de las constantes del pensamiento político liberal es la insistencia en exigir al Estado neutralidad frente a las concepciones éticas que pueden sustentar las personas. Se diría que una concepción así no puede abogar por ninguna tradición ética en particular. La indiferencia que el liberalismo parece mostrar frente a las concepciones que la gente puede tener sobre la felicidad y la vida buena no parece, sin embargo, concordar con el magnetismo y el vigor que esta

\footnotetext{
${ }^{26}$ Una aclaración terminológica. En el lenguaje corriente, tendemos a utilizar "ética” y "moral” como vocablos intercambiables. Dworkin, siguiendo un uso bastante extendido ya en el lenguaje especializado de la filosofía moral, los utiliza como términos técnicos de forma diferenciada. Por "ética" entiende convicciones que permiten juzgar sobre la bondad de la vida que uno puede decidir vivir, mientras que por "moral" entiende un conjunto de normas o principios que orientan el modo como alguien debe tratar a terceros. Diciéndolo simplificadamente, en Dworkin la ética encarna el reino de "lo bueno" y la moral el reino de "lo justo" o de "lo debido".
} 
ideología ha demostrado en el pasado, y que parece que vuelve a demostrar apenas nos detenemos a reflexionar sobre algunos de los acontecimientos más relevantes que ha protagonizado la historia política mundial más reciente. Como afirma el autor, la tradición liberal "tiene por fuerza que tener raíces en imágenes muy poderosas acerca de lo que es posible y bueno para la vida de la gente" 27 .

El liberalismo, que es una tradición política que propugna entre sus ideales fundamentales el ideal de la tolerancia, se enfrenta así al reto de tener que explicar qué razón o razones se supone que puede exhibir la gente para abrazar un proyecto político de ese tipo como parte integrante de una forma de vida valiosa.

Llegados a este punto, la estrategia del autor se desarrolla en dos pasos. En primer lugar, se pone a la tarea de diseñar una concepción ética (que bautiza como "individualismo ético" ${ }^{28}$ ) sobre la que asentar su proyecto filosófico-político, para, a continuación, mostrar de qué forma éste fluye de aquélla.

No me detendré en el examen del individualismo ético, pues considero que identifica y organiza un conjunto de valores éticos ampliamente compartidos que pueden ser aceptados por todos, a pesar de los desacuerdos sobre temas éticos y religiosos más concretos que persistirían entre nosotros. Más bien, me pararé, siquiera brevemente, en la "estrategia" propiamente de fundamentación que ensaya el autor.

Las sociedades democráticas modernas, profundamente divididas ética y religiosamente y en otras dimensiones valorativas, tienen así que resolver el problema de reconciliar a personas con concepciones diferentes de la vida buena, de modo que sin embargo se mantenga la unidad social. "Continuidad" y "discontinuidad" designan dos estrategias diferentes que puede seguir el liberalismo para ofrecerse como una concepción capaz de conseguir la reconciliación entre ética y política.

En la estrategia de la discontinuidad, el liberalismo se concibe a sí mismo y presenta públicamente como una construcción en cierto sentido artificial, diseñada con el solo objeto de servir a la discusión pública. Pero si está pensado solo como una concepción política, orientada a la discusión política, entonces no hay necesidad de que nadie suscriba sus contenidos o principios como "suyos", con lo cual

27 Dworkin, Ética privada e igualitarismo político, Barcelona, Paidós, 1993, p. 43.

${ }^{28}$ Dworkin, «In Praise of Theory», Arizona State Law Journal, 29, 1997, p. 19. El individualismo ético es el nombre que elige Dworkin para designar una teoría ética construida sobre la base de dos grandes principios éticos: el principio de igual importancia y el principio de especial responsabilidad. El principio de igual importancia establece que una vez que ha comenzado una vida es importante desde un punto de vista objetivo que no se eche a perder, y que eso es igualmente verdadero respecto de cualquier otra vida humana. El principio de especial responsabilidad establece que sobre cada persona pesa una responsabilidad especial y personalísima de hacer de su vida una vida valiosa. Esos dos principios contendrían un desarrollo particular de dos de los tres grandes grupos de temas éticos esenciales que acabamos de formular: el primer principio ofrece una respuesta a las cuestiones relativas a la fuente, mientras que el segundo, en cambio, ofrece respuesta a las cuestiones relativas a la responsabilidad. 
ética y política resultan finalmente compatibles entre sí. Precisamente el hecho de que se presente independiente de presupuestos éticos permite que puedan abrazarla personas con concepciones éticas diferentes. La tradición contractualista, y de modo particular la teoría de Rawls ${ }^{29}$, representarían a juicio de Dworkin ejemplos importantes de esta estrategia.

La estrategia de la continuidad intenta reconciliar ética y política presentando los valores políticos del liberalismo como derivados de valores éticos más fundamentales: persigue una experiencia moral más integrada, y en ese sentido se muestra reticente a dejar de lado las convicciones éticas cada vez que hay que entrar a discutir un asunto político. Mientras que en el primer caso el liberalismo, como teoría política, no se levanta sobre una ética específicamente liberal, sino que aspira a poder ser compatible con una cierta variedad de concepciones éticas, cuando adopta la estrategia de continuidad intenta construir una ética que sea capaz de congeniar con los principios políticos liberales —en suma, una ética liberal一. Si decíamos hace un momento que el liberalismo político de Rawls se puede interpretar como un exponente descollante de la estrategia de discontinuidad, la igualdad liberal de Dworkin aspira a convertirse en paradigma de la estrategia de continuidad ilustrando una nueva concepción o forma de entender el liberalismo como liberalismo ético ${ }^{30}$.

Según Dworkin la estrategia de la discontinuidad está desencaminada. El motivo es que, aplicada consistentemente, parece condenada a crear un conflicto berliniano entre los valores éticos y los políticos: se propone resolver el desacuerdo que pueda haber entre las personas a propósito de los valores éticos a costa de un conflicto en cada persona entre sus diversos valores. Pero con ello se condena a los ciudadanos a una existencia dividida.

Ahora bien, la estrategia de la continuidad propuesta por Dworkin parece, por su parte, enfrentarse a una objeción: si admitimos que las concepciones comprehensivas de la vida buena son irremediablemente particulares (incluso cuando son compartidas por muchos), un liberalismo que se presente públicamente como una concepción ética ¿̇no se está arrebatando a sí mismo la posibilidad de focalizar un consenso inclusivo? A decir verdad, se diría que liberalismo político y liberalismo ético son respuestas complementarias, e insatisfactorias, a lo que el mismo Dworkin ha denominado el «dilema consensual ${ }^{31}$ : si el liberalismo político, en la línea de

\footnotetext{
29 Rawls, political Liberalism, Nueva York, Columbia University Press, I993.

30 «Cuando elegimos entre contrato y continuidad, como estrategias para arraigar la política liberal en la ética personal, estamos eligiendo también entre dos formas de liberalismo" (Dworkin, Ética privada e igualitarismo politico, op. cit., p. 66).

31 Dworkin, «Justice for Hedgehogs» (inédito), I999, p. 8.
} 
Rawls, paga el consenso al precio del desdoblamiento y el conflicto, el liberalismo ético dworkiniano paga la integridad al preciso del consenso.

Dworkin minimiza este inconveniente. Para empezar, sugiere que la estrategia rawlsiana del consenso tropieza con serios problemas de aplicabilidad si, como parece sugerir la experiencia, la gente no está dispuesta a preterir sus principios, instintos y convicciones éticas y religiosas más básicas a favor de un conjunto discreto de principios políticos. Por otra parte, Dworkin cree que, a pesar del atractivo que tiene la idea de consenso, el consenso no es necesario a efectos legitimatorios: «si la estructura política en la que persiste el acuerdo es verdaderamente democrática —es decir, si realmente es una asociación (partnership) entre iguales [...] - entonces es equitativo (fair) imponer sus decisiones colectivas incluso a aquéllos que se oponen a ellas, e incluso a aquéllos que erróneamente piensan que la estructura del gobierno es injusta [...] No nos enfrentamos a un dilema consensual sino a un dilema democrático: no necesitamos consenso, sino popularidad (popularity), al menos popularidad potencial ${ }^{32}$ ».

Así las cosas, ¿la versión ética del liberalismo auspiciada por Dworkin resulta superior a la versión política representada por Rawls? Mi opinión es que estamos ante dos estrategias complementarias. En realidad, "liberalismo político" y "liberalismo ético" son dos formas diferentes de reacción del pensamiento liberal al desafío que supone el pluralismo para las sociedades modernas: mientras Rawls quiere presentar este ideario político como una concepción exclusivamente política, independiente de concepciones filosóficas, morales y éticas comprehensivas, con el fin de convertirlo en el foco de un consenso constitucional entre personas con concepciones diferentes de la vida buena, Dworkin busca atraer adeptos al liberalismo mostrando por el contrario que descansa en una concepción atractiva de la vida buena. En un mundo en el que se ha instalado irreversiblemente el hecho del pluralismo, Rawls aspira a que los principios políticos liberales puedan atraer un consenso constitucional, mientras que Dworkin pretende que estos aumenten su popularidad frente a otras concepciones rivales que se disputan la adhesión en el universo cultural de una sociedad pluralista. Pero no son dos estrategias incompatibles. En realidad, desde el punto de vista de Rawls, el liberalismo ético de Dworkin puede integrarse sin dificultad, igual que otras concepciones comprehensivas [liberales y no liberales], en el "consenso por solapamiento» en que descansa la unidad constitucional en un mundo pluralista.

Por lo demás, frente a la acusación de Dworkin de que Rawls propicia una estrategia esquizoide de discontinuidad entre principios políticos y convicciones

32 Dworkin, "Justice for Hedgehogs», op. cit., pp. 9-10. 
éticas, creo que en un mundo pluralista sólo un planteamiento "político" al estilo del propiciado por Rawls hace compatible la unidad social y la continuidad entre las diversas convicciones éticas comprehensivas y los principios político-constitucionales. A mayor abundamiento, no creo que sea verdad que el enfoque rawlsiano imponga, tal y como sugiere Dworkin, discontinuidad esquizofrénica entre ética y política. En realidad el enfoque político rawlsiano articula "continuidad" y "discontinuidad": aunque los principios no descansan en una concepción comprehensiva, el consenso por solapamiento implica que cada uno de los que participa en él lo hace a partir de sus propias concepciones que, si son razonables, le inducirán a aceptar los principios de la razón pública cuando se discute sobre cuestiones constitucionales básicas. En este sentido el liberalismo ético de Dworkin puede integrarse sin dificultades en el liberalismo político de Rawls. Por otra parte, hay que señalar que también en Dworkin se presenta en cierto sentido esta combinación: en la medida en que los principios del individualismo ético y el modelo del desafío son altamente abstractos, y entre ellos y la concepción de la igualdad liberal no hay una relación fuerte de derivación, estos admiten una cierta variedad de concreciones políticas. 


\section{REFERENCIAS BibLIOGRÁficas}

Beruin, I., The Hedgehog and the Fox, Weidenfeld and Nicolson Ldt., 1953.

Dworkin, R., Taking Rights Seriously, London, Duckworth, 1981.

- A Matter of Principle, Londres, Harvard University Press, 1985.

- Law's Empire, London, Fontana Press, 1986.

— «Equality, Democracy, and Constitution: We the People in Court», Alberta Law Review, 28, 1990, 324-346.

- Ética privada e igualitarismo político, Barcelona, Paidós, 1993.

— «Constitutionalism and Democracy», European Journal of Philosophy, 3, 1995, 2-11. DOI: https://doi.org/10.1111/j.1468-0378.1995.tb00035.x

- Freedom's Law. The Moral Reading of American Constitution, Oxford, Oxford University Press, 1996.

— «In Praise of Theory», Arizona State Law Journal, 29, 1997, 353-376.

— "The Partnership Conception of Democracy», California Law Review, 86, 1998, 453-458. DOI: https://doi.org/10.15779/Z386B0W

— «ustice for Hedgehogs» (inédito), 1999.

- Sovereign Virtue. The Theory and Practice of Equality, Cambridge, Mass., Harvard University Press, 2000.

Rawls, J., Political Liberalism, Nueva York, Columbia University Press, 1993.

Raz, J., Practical Reason and Norms, Princeton, Princeton University Press, 1990.

DOI: http://doi.org/10.15366/bp2019.20.005

Bajo Palabra. II Época. No20. Pgs: 87-106 
